\title{
Labyrinthe
}

$7 \mid 2000$

Numéro 7

\section{La revue Preuves (1951-1974) : l'expression d'une intelligentsia dans le champ anticommuniste}

\section{Benjamin Achab}

\section{(2) OpenEdition}

12 Journals

Édition électronique

URL : http://journals.openedition.org/labyrinthe/733

DOI : $10.4000 /$ labyrinthe.733

ISSN : 1950-6031

Éditeur

Hermann

Édition imprimée

Date de publication : 30 octobre 2000

Pagination : 166-168

Référence électronique

Benjamin Achab, «La revue Preuves (1951-1974) : I'expression d'une intelligentsia dans le champ anticommuniste », Labyrinthe [En ligne], 7| 2000, mis en ligne le 20 avril 2005, consulté le 22 avril 2019. URL : http://journals.openedition.org/labyrinthe/733 ; DOI : 10.4000/labyrinthe.733

Ce document a été généré automatiquement le 22 avril 2019

Propriété intellectuelle 


\title{
La revue Preuves (1951-1974) : l'expression d'une intelligentsia dans le champ anticommuniste
}

\author{
Benjamin Achab
}

1 L'étude menée ici vise à mieux connaître l'anticommunisme des intellectuels pour la seconde moitié du siècle*. Celui-ci fut longtemps prisonnier de l'opprobre jeté par les intellectuels dans la mouvance du P.C.F. À l'aide des articles et manifestes, il s'agit d'esquisser une typologie des grands courants de l'anticommunisme présents au sein de la revue Preuves et, par extension, de celui des intellectuels en général. Par ailleurs, on tente d'apprécier son influence au gré des crises survenues à l'Est et notamment de la crise hongroise de 1956. L'interrogation se poursuit afin de déterminer sa place dans le champ anticommuniste et pour savoir si au-delà des tendances politiques, il existe un ciment idéologique propre à l'anticommunisme en général.

2 La naissance de la revue Preuves ${ }^{1}$, en 1951, s'effectue dans le cadre des tensions intellectuelles et politiques de la guerre froide. Face au Kominform, les États-Unis soutiennent l'entreprise dirigée par François Bondy. Cet ancien membre du P.C.F. en est le fondateur. Une première strate regroupe alors la gauche avec notamment Maximilien Rubel qui préside à ce que l'on peut appeler un anticommunisme de différenciation. Il détache la pensée de Marx de l'édification de l'U.R.S.S. Un de ses articles, intitulé « Karl Marx, auteur maudit en U.R.S.S. ", sert à démystifier chez les communistes staliniens une vision issue de la propagande faisant de Staline « le génial continuateur de Marx ». Il est finalement davantage question d'anti-stalinisme. Une autre tendance à gauche consiste à combattre le communisme mythique, en lui substituant un socialisme désacralisé (Gilles Martinet). La seconde strate est formée d'une droite libérale menée par Raymond Aron. Ce dernier développe à l'opposé de Rubel une critique englobante ou procédé d'amalgame mêlant dans une même famille Marx, marxisme et U.R.S.S. À la mythologie communiste répond donc une certaine mythologie anticommuniste issue du courant libéral. La droite extrémiste, quant à elle, dénonce "la face de méduse du communisme " (Thierry Maulnier) et joue sur la peur d'un Occident menacé par la barbarie communiste. Cette 
seconde strate prend de l'ampleur pour succéder, à partir de 1956, à la ligne socialdémocrate. À ces deux strates, il convient d'ajouter la contribution clef des excommunistes, rendue intelligible à l'aide du concept de génération.

3 Partant de l'axe de l'anticommunisme ${ }^{2}$, on perçoit trois générations de combat qui diffèrent par un cadre de formation politique modifié, eu égard à l'évolution de l'histoire de l'U.R.S.S. La génération «17» qui recèle la charge d'illusion la plus lourde et la connaissance la plus fine du communisme va se trouver, face à la dégénérescence de la révolution russe d'Octobre, confrontée à un véritable processus de "dissonance cognitive ». C'est le cas de Souvarine, haut responsable du Komintern. Toutefois, une lecture intra-générationelle révèle l'importance du ressort comportemental. Face à un même événement, les trajectoires diffèrent. Souvarine, par exemple, à la différence de Silone, (ancien membre du P.C. italien, qui se détache simplement de l'idéologie) apporte sa collaboration à l'anticommunisme d'État. À des degrés divers, ce schéma reste valable pour les « ex- » des années 30 comme Koestler ou Sperber. Puis, après la répression de l'insurrection hongroise de 1956 et un temps d'incubation post-communiste, parviennent les retournements d'Annie Kriegel et de François Furet qui collaborent à la revue. L'anticommunisme crée donc une solidarité au-delà des courants politiques.

Une démarche active et avant-gardiste caractérise aussi Preuves. Jelenski fait la promotion de la dissidence polonaise en publiant Gombrowicz et Milosz. La revue œuvre encore, à l'aide de témoignages sur les camps soviétiques, pour la stigmatisation du totalitarisme. Ses propres relais d'édition, les conseils de lecture ou des encadrés pour «Paix et Liberté " (l'association de J.-P. David, d'un anticommunisme virulent), aident à la diffusion d'une culture propre et révèlent un champ anticommuniste fait de ramifications multiples. Mais, à partir de 1966, s'amorce le déclin consécutif à la révélation du financement de Preuves par la C.I.A. La revue disparaît alors en 1974 et certains de ses auteurs poursuivent ailleurs leur combat anticommuniste.

5 Au terme de l'étude, Preuves, mensuel tiré à 10000 exemplaires et regroupant un vaste panel politique, puise sa force, dès les origines, dans la contribution d'anciens communistes. Bien que son influence soit limitée car l'anticommunisme se trouve alors minoritaire, elle marque durablement, par le débat qu'elle suscite ainsi que par sa connaissance de l'univers communiste.

Aujourd'hui, suite à la chute de l'U.R.S.S., l'anticommunisme, dégagé du rejet de la communauté intellectuelle, reprend l'ascendant. Mais il verse encore dans la caricature, au mépris d'une approche qui devrait mieux lier théorie et pratique ${ }^{3}$.

\section{NOTES}

*. Synthèse du mémoire de maîtrise soutenu en 1997 sous la direction de M. Robert Vandenbussche à l'Université Charles de Gaulle-Lille III.

1. Pierre Grémion, Preuves, une revue européenne à Paris (Anthologie), Paris, Julliard/ Commentaire, 1989. 
2. Pour une première approche, on consultera avec profit la synthèse de J.-J. Becker et $\mathrm{S}$. Berstein, Histoire de l'anticommunisme en France, 1900-1940, Paris, Orban, 1987.

3. Le parallèle communisme-nazisme notamment, envisagé de manière globale, apparaît très discutable. Par ailleurs, on s'interroge encore insuffisamment sur la filiation MarxLénine-Staline, qui produirait sans doute un isolat de Marx vis à vis de ses successeur,s ainsi que sur la dichotomie théorie-pratique. 